\title{
Sodium Bicarbonate-Based Warm Saline Versus Warm Saline for Peritoneal Irrigation in Diagnostic Hysteroscopy-Laparoscopy: A Prospective Randomized Controlled Trial \\ Mohamed adel aboelela \\ Anesthesia and Surgical Intensive Care, Mansoura University. \\ Corresponding author: Mohamed Adel Aboelela, email:aboelela_mohamed@yahoo.com, ORCID: https://orcid.org/0000-0001-8019-0243, Mobile: 01061546753
}

\begin{abstract}
Introduction: Diagnostic hysteroscopy-laparoscopy is an essential tool in management of infertility. Mainly, postoperative pain related to insufflated cold carbon dioxide $\left(\mathrm{Co}_{2}\right)$, as it creates acidic milieu causing mucosal, subdiaphragmatic and phrenic nerve irritation. Sodium bicarbonate is an alkaline solution, neutralizing the acidic effects, hence reduces postoperative pain and irritation symptoms.

Objectives: This study aimed to assess the efficacy of adding sodium bicarbonate to washing warm saline in hysteroscopic-laparoscopic patients regarding incidence and intensity of postoperative pain and gastrointestinal irritation symptoms.

Patients and methods: 66 patients were enrolled, one was excluded due to ovarian bleeding. 33 patients in sodium bicarbonate $(\mathrm{Na})$ group received $50 \mathrm{ml}$ sodium bicarbonate $8.4 \%$ in $1000 \mathrm{ml}$ warm saline as peritoneal irrigation solution. While 32 patients in saline (S) group the peritoneal irrigation solution was $1000 \mathrm{ml}$ warm saline only.

Results: Patients' demographic, hemodynamic data were comparable. Postoperative pain profile regarding shoulder tip pain, visual analogue score (VAS), and total pethidine consumption were lower in Na group. Also, postoperative nausea and vomiting (PONV) was lower in Na group.

Conclusion: Adding sodium bicarbonate to warm saline as a peritoneal washing solution during hysteroscopylaparoscopy procedure resulted in better postoperative pain reduction specially shoulder tip pain and reduced the incidence of PONV. These results would improve patients' recovery and experience towards operative practice.
\end{abstract}

Keywords: Laparoscopy, Pain, VAS, Sodium bicarbonate, PONV.

\section{INTRODUCTION}

Infertility is a common health problem affecting nearly $10 \%$ of women at reproductive age. The hysteroscopy and laparoscopy are becoming very important in the management of such pathology ${ }^{(1)}$. While, it seems simple procedure, postoperative pain is an agonizing complication ${ }^{(2)}$.

Pneumoperitoneum and insufflated cold carbon dioxide create gas pockets, beside acidic milieu, mucosal irritation and phrenic nerve damage. All of these factors are associated with postoperative visceral and shoulder tip pain ${ }^{(3,4)}$. Many trials investigated different ways to reduce such pain. Systemic analgesic drugs, local analgesia, peritoneal wash, regional blocks and gas washing techniques had been prescribed, with variable results and also, side effects ${ }^{(5-9)}$.

Therefore, the clinical significance of pain control after laparoscopic surgery remains controversial. Sodium bicarbonate has a neutralizing effect against the acid milieu of the peritoneal cavity and the phrenic nerve damage. Also, warm saline counteracts the cold effect of carbon dioxide, which consecutively will lead to a reduction of post laparoscopic pain ${ }^{(10)}$.

In this trial, we assessed efficacy of adding sodium bicarbonate to washing warm saline in hysteroscopiclaparoscopic patients regarding incidence and intensity of postoperative pain and gastrointestinal irritation symptoms.

\section{PATIENTS AND METHODS}

This study was conducted at Mansoura University Hospital. Sixty six patients were enrolled in this study, which adheres to the applicable CONSORT guidelines (figure 1).

\section{Ethical approval:}

This study was approved by the Institutional Review Board, Mansoura Faculty of Medicine (IRB \# R.20.02.737, February 15-2020). Informed consents were obtained from all subjects participating in the trial. Before patients' enrolment, the trial was registered in the Pan African clinical trial registry (PACTR202003752686494, date of registration: March 2-2020). Included females were ASA I or II aging 18-45 years, scheduled for diagnostic hysteroscopic-laparoscopic procedures.

Exclusion criteria: Patient refusal, allergy to used drugs, addiction, altered liver or kidney function, psychological troubles or complicated procedure. Candidate patients were randomly allocated into two groups [33 for saline group (S group), 33 for sodium bicarbonate group ( $\mathrm{Na}$ group)] by random number 
generator of software program with closed envelop technique received with patients file.

Patients had preoperative assessment for anesthetic fitness and matching the inclusion and the exclusion criteria. At the operative suite, patients received pantoprazole (Zurcal $40 \mathrm{mg}$, AUG pharma, Spain), dexamethasone (Dexamethasone, Sigmatic, Egypt) 8 $\mathrm{mg}$ and $3 \mathrm{mg}$ of midazolam (Midathetic, 1044mount pharmaceuticals).

In operating room, patients were connected to basic monitor including ECG, NIBP and pulse oximeter. Anesthesia was induced using propofol $1-2 \mathrm{mg} / \mathrm{kg}^{-1}$ (Diprivan, Fresenius KABI), Fentanyl $1 \mu / \mathrm{kg}^{-1}$ (fentanyl Hameln, Hameln pharmaceuticals, Germany), Atracurium $0.6 \mathrm{mg} / \mathrm{kg}^{-1}$ (Atrabesylate, Egypharm, Egypt). A proper sized endo-tracheal tube was inserted and fixed in place after confirmation of correct positioning. Patients were ventilated using volume controlled ventilation mode to keep $\mathrm{EtCO}^{2}$ between 33$35 \mathrm{mmHg}$. Anesthesia was maintained using sevoflurane $1-2 \%$ in $40 \%$ oxygen-air gas mixture. Topup dose of atracurium was given on needs and Ringer acetate infusion at rate $4 \mathrm{ml} / \mathrm{kg}^{-1} / \mathrm{h}^{-}$was started in a suitable peripheral intravenous line.

Surgeon was allowed to start hysteroscopic examination then start abdominal insufflation with carbon dioxide slowly at a rate $1-1.5 \mathrm{l} / \mathrm{min}$ with maximum insufflating pressure $13-15 \mathrm{mmhg}$ under close hemodynamics observation by the researcher anesthesiologist.

According to randomization received in the closed envelop with the patient file, wash out solution used in peritoneal irrigation prepared by independent anesthesia technician as $1000 \mathrm{ml}$ warm saline in (S) group and $50 \mathrm{ml}$ sodium bicarbonate $8.4 \%$ added to $1000 \mathrm{ml}$ warm saline in sodium bicarbonate group (Na group). Surgeon was allowed to use the wash solution during the procedure, while both surgeon and researcher anesthesiologist were blinded of its content. After completion of the procedure, patients were kept in supine position. Surgeon was asked to ensure exsufflation by doing gentle abdominal pressure, remove the trocars and infiltrate trocars site by $10 \mathrm{ml}$ lidocaine $2 \%$ to relief pain. Reversal of muscle relaxation was done using neostgmin $0.05 \mathrm{mg} / \mathrm{kg}^{-1}+$ atropine $0.02 \mathrm{mg} / \mathrm{kg}^{-1}$, and awake extubation after fulfillment of extubation criteria. Postoperative analgesia based on $1 \mathrm{gm}$ of paracetamol every 8 hours, rescue analgesia used pethidine $0.5 \mathrm{mg} / \mathrm{kg}^{-1} \mathrm{IV}$ when VAS $\geq 4$.

Collected data included postoperative shoulder tip pain at 1, 6, 12 and 24 hours postoperatively as a primary outcome objective. Other data included overall pain assessment using VAS score, heart rate (HR), mean arterial blood pressure (MAP) immediate after recovery and at 1, 2, 4, 6, 12 and 24 hours as well as total pethidine consumption and incidence of postoperative nausea and vomiting within 24 hour. All postoperative data were recorded by trained nurses, who were blinded to intervention techniques.

In this trial, we hypothesized that adding sodium bicarbonate to warm saline for peritoneal irrigation will improve postoperative pain control, reduce gastrointestinal irritation symptoms with stable hemodynamics.

\section{Sample size Statistical analysis and data collection}

The incidence of shoulder tip pain after laparoscopic gynecologic surgery ranged from $30 \%$ to $80 \%$ (4). Using a $60 \%$ incidence and assuming a reduction of $40 \%$ when sodium bicarbonate is used for peritoneal irrigation, the required sample size to achieve a study power of 0.8 with an alpha error of 0.05 was 56 cases. Additional 10 cases were added to compensate for dropouts, with total enrolment of 66 cases. G*power software version 3.1.9.4 was used for sample size calculation.

Perioperative data will be tabulated and analyzed using IBM SPSS software version 22. Continuous data were presented as mean \pm SD or median IQR according to normality of distribution. Nominal and categorical data were presented as numbers and percentages. Independent sample $\mathrm{T}$ test, Mann-Whitney test or chi square test were utilized to detect statistical differences between the studied groups.

\section{RESULTS}

This study enrolled 66 patients scheduled for diagnostic hysteroscopy-laparoscopy, one patient was excluded from intervention due to ovarian bleeding during ovarian drilling, presented in figure (1). Patients' demographics, basal hemodynamic data, and duration of anesthesia are presented in table (1) without significant difference between the studied groups.

Postoperative hemodynamic measurements (HR, MAP) are illustrated in figure (2), without significant difference between the studied groups.

Postoperative patients' characteristics are illustrated in table (2), shoulder tip pain was significantly lower in Na group at 1, 6 and 12 postoperative hours $(\mathrm{P}$ value $0.001,0.001,0.001$ consecutively). Total pethidine consumption was significantly lower in Na group (P value 0.041 ), and PONV was significantly lower in NA group (P value 0.007).

Postoperative VAS score is presenting in table (3), VAS score was lower in $\mathrm{Na}$ group immediate postoperative, and at 1,2 and 4 hours postoperatively ( $\mathrm{P}$ value, $0.001,0.001,0.001,0.003$ consecutively), while it was comparable among both groups at other times of assessment.

None of the studied patients showed any side effects or unanticipated complications related to techniques used. 
Table (1): Patients' demographic data, basal hemodynamic data, and duration of anesthesia

\begin{tabular}{llll} 
& Group S & Group NA & $P$ \\
\hline Age (years) & $30.68 \pm 2.83$ & $29.45 \pm 4.98$ & 0.2 \\
BMI (kg/m2) & $23.13 \pm 1.59$ & $23.42 \pm 1.97$ & 0.5 \\
Basal HR (bpm) & $79.81 \pm 5.60$ & $81.54 \pm 6.57$ & 0.3 \\
Basal MAP (mmHg) & $90.43 \pm 4.36$ & $90.03 \pm 5.45$ & 0.7 \\
Duration of anesthesia (minutes) & $57.06 \pm 4.88$ & $55.96 \pm 4.68$ & 0.4 \\
\hline
\end{tabular}

BMI: body mass index, HR: heart rate, MAP: mean arterial blood pressure. ${ }^{* P}$ value is significant if less than 0.05

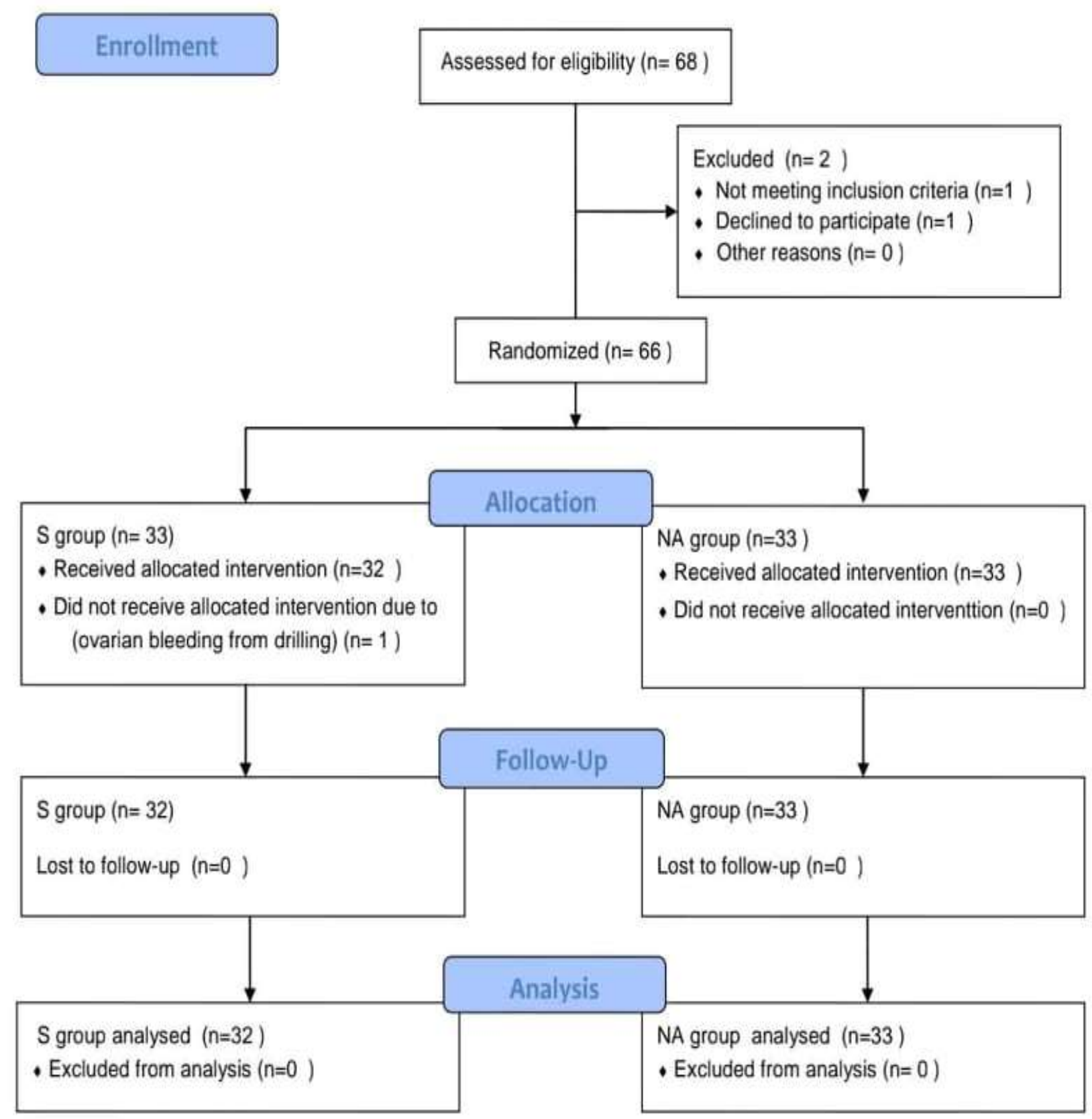

Figure (1): Consort flow diagram for the study 


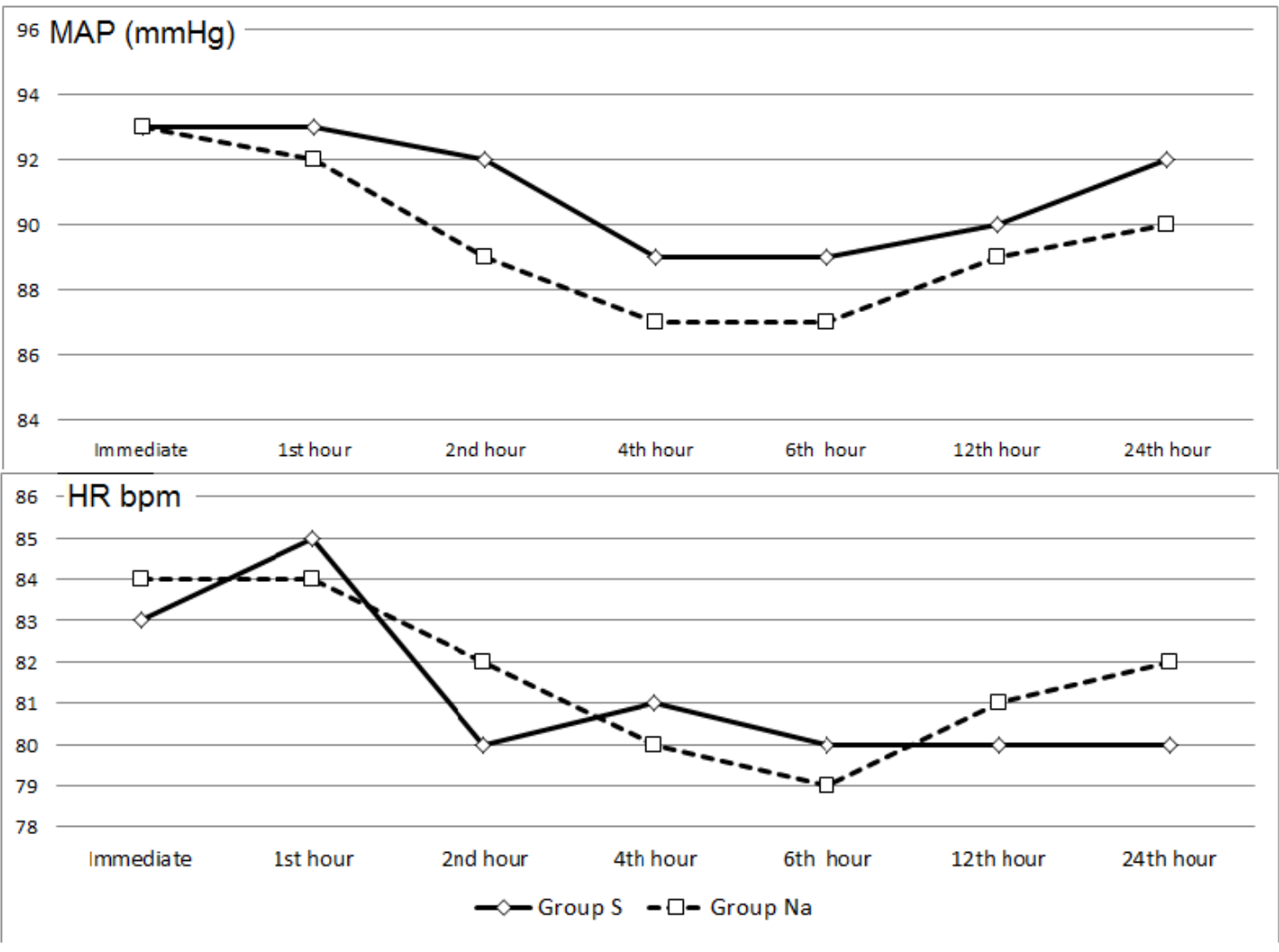

Figure (2): Postoperative hemodynamics data (HR \& MAP) of the included patients

Table 2: Postoperative data for the study groups. Data are presented as numbers (percentage \%), median (interquartile range).

\begin{tabular}{llll}
\hline & Group S & Group NA & $P$ \\
\hline Pethidine consumption & $80.0(62.5)$ & $40.0(47.5)$ & $0.041^{*}$ \\
PONV & $12(37 \%)$ & $3(9 \%)$ & $0.007^{*}$ \\
Shoulder tip pain & & & \\
-1 hour & $21(66 \%)$ & $8(24 \%)$ & $0.001^{*}$ \\
-6 hours & $23(72 \%)$ & $7(21 \%)$ & $0.001^{*}$ \\
-12 hours & $13(41 \%)$ & $2(6 \%)$ & $0.001^{*}$ \\
-24 hours & $4(12 \%)$ & $0(0 \%)$ & 0.053 \\
\hline
\end{tabular}

PONV: postoperative nausea and vomiting. $\quad * P$ value is significant if less than 0.05 
Table 3: Postoperative visual analogue score (VAS) for the study groups. Data are presented as median (interquartile range).

\begin{tabular}{llll}
\hline & Group S & Group NA & $P$ \\
\hline VAS0 & $5(2)$ & $3(2)$ & $0.001^{*}$ \\
VAS 1h & $4(1)$ & $3(1)$ & $0.001^{*}$ \\
VAS 2h & $3(1)$ & $2(1)$ & $0.001^{*}$ \\
VAS 4h & $2(1)$ & $2(0)$ & $0.003 *$ \\
VAS 6h & $2(1)$ & $1(1)$ & 0.077 \\
VAS 12h & $1(2)$ & $1(1.5)$ & 0.727 \\
VAS 24h & $0(1)$ & $0(1)$ & 0.693 \\
\hline
\end{tabular}

VAS: visual analogue score.

$* P$ value is significant if less than 0.05

\section{DISCUSSION}

This study investigated the effect of adding sodium bicarbonate to warm saline as irrigation fluids during peritoneal wash in diagnostic hysteroscopylaparoscopy procedure, and its impact on postoperative pain. Our results showed that the incidence of postoperative shoulder tip pain was significantly lower in $\mathrm{Na}$ group at the first 12 postoperative hours. Also, VAS score was significantly lower in $\mathrm{Na}$ group at the first 4 postoperative hours, rescue analgesia consumption was significantly higher in S group, and overall incidence of PONV during the first postoperative 24 hours was higher in $S$ group. Meanwhile, hemodynamics were comparable in both groups.

The hysteroscopy-laparoscopy is a simple and essential tool in diagnosis of infertility, it can reveal the presence of peritubal adhesions, periadnexal adhesions, tubal pathology, and endometriosis in 35$68 \%$ of cases even after normal hystero-salpingogram result ${ }^{(11)}$. Hence, it is a non-traumatic surgery, the insufflated cold carbon dioxide to create pneumoperitoneium for laparoscopy accounts for post laparoscopy pain. Coldness, acidic milieu and formed gas pockets lead to continuous mucosal, subdiaphragmatic and phrenic nerve irritation with consecutive visceral and shoulder tip pain during postoperative times ${ }^{(12-14)}$. Strategies applied to reduce such pain include local and systemic analgesic drugs, regional blocks, peritoneal irrigation, pulmonary recruitment maneuver and using humidified insuflating gases ${ }^{(5-9,15-18)}$.

According to our results, using of sodium bicarbonate with warm saline in peritoneal irrigation during laparoscopy resulted in better postoperative pain profile with less gastro-intestinal irritation symptoms. This can be explained by counteracting effect to acidic milieu and cold effect of insufflated gas during the procedure. Sodium bicarbonate is an alkaline solution, used to neutralize $\mathrm{PH}$ in systemic acidity conditions when used by intravenous route ${ }^{(19}$, 20). Also, it can counteract local acidity effect of injected local anesthesia drugs when used by subcutaneous route ${ }^{(2-23)}$. Based on this idea, sodium bicarbonate neutralized the acidic effect created by insufflated carbon dioxide when used with saline for peritoneal irrigation. Also, the used warm saline in both groups seems at decreasing the draw backs of cold carbon dioxide. As insufflating dry and cold $\mathrm{CO}_{2}$ into the abdomen causes peritoneal damage, post-operative pain, hypothermia and post-operative adhesions ${ }^{(24)}$. Using both sodium bicarbonate and warm irrigation fluid create a more physiologic peritoneal environment and reducing the post- 
operative pain and gastro-intestinal irritation symptoms. These results of pain reduction are important in improving patients' recovery and overall patients experience towards the operative practice, specially in such group of patients. Also, the reduction in rescue analgesia use will shorten the hospital stay, with less possibility of incidence of hospital stay complications, and less economic cost.

Saadati K. et al. ${ }^{(10)}$ used sodium bicarbonate in peritoneal irrigation with laparoscopic cholecystectomy patients. They concluded that, intraperitoneal irrigation of sodium bicarbonate is a simple and safe method that provides pain relief in the postoperative period after laparoscopic cholecystectomy compared with none-washing. Also, intraperitoneal irrigation of sodium bicarbonate was found to have a better pain relief profile compared with normal saline. Also, the trials of peritoneal irrigation with normal saline resulted in better carbon dioxide wash with consecutive better postoperative pain profile ${ }^{(25-27)}$.

Regarding PONV, acidosis and tissue hypoxia increase its risk. Maneuvers to decrease these effects associated with better postoperative results with less peritoneal changes and gastro-intestinal irritation symptoms (28). No unanticipated side effects or complications could be detected regarding both techniques.

Our study had some limitations; we did not detect intra-abdominal pressure, only depended on insufflating pressure. Also, we did not detect inflammatory mediators. The use of inflammatory mediators would add to the strength of the study. Also, the limited time for postoperative monitoring, only for 24 hours, may have concealed some clinical differences between the studied techniques. Larger scale studies with measurement of intra-abdominal pressure, and inflammatory mediators, and longer patient follow-up periods are recommended.

\section{CONCLUSION}

Post laparoscopy pain is an agonizing complication. Many trials studied different techniques in management of such pain. Sodium bicarbonate is an alkaline solution that neutralize acidity of insufflated carbon dioxide. This will lead to reduction of overall postoperative pain sensation and postoperative gastro-intestinal irritation symptoms.

\section{ACKNOWLEDGMENT}

For staff nurses in operating room and gynecological ward, Mansoura University Hospital, Egypt, who provided high quality perioperative care for our patients.

\section{REFERENCES}

1.Boudhraa K, Jellouli M, Kassaoui O et al. (2009): Role of the hysteroscopy and laparoscopy in management of the female infertility: about 200 cases. Tunis Medicine, 87 (1): $55-60$.

2.Zeeni C, Chamsy D, Khalil A et al. (2020): Effect of postoperative Trendelenburg position on shoulder pain after gynecological laparoscopic procedures: a randomized clinical trial. BMC Anesthesiol., 29 (1): 207.

3.Chung J, Kang K, Park S et al. (2017): Effect of intraperitoneal CO 2 concentration on postoperative pain after laparoscopic cholecystectomy. Annals of Surgical Treatment and Research Effect, 93: 181-5.

4. Kaloo P, Armstrong S, Kaloo C et al. (2019): Interventions to reduce shoulder pain following gynaecological laparoscopic procedures. doi: $10.1002 / 14651858$.

5. Alkhamesi N, Peck D, Lomax D et al. (2007): Intraperitoneal aerosolization of bupivacaine reduces postoperative pain in laparoscopic surgery: a randomized prospective controlled double-blinded clinical trial. Surg Endoscopy, 21: 602-6.

6. Kendall M (2018):Intraoperative analgesic regimen in laparoscopic bariatric surgery: a closer look. Surgery for Obesity and Related Diseases, 14 (6): 876.

7.Kucuk C, Kadiogullari N, Canoler O et al. (2007):A placebo-controlled comparison of bupivacaine and ropivacaine instillation for preventing postoperative pain after laparoscopic cholecystectomy. Surg Today, 37: 396400.

8.Erol D, Yilmaz S, Polat C et al. (2008):Efficacy of thoracic epidural analgesia for laparoscopic cholecystectomy. Adv Ther., 25 (1): 45-52.

9.Khan K, Khan R (2018): Analgesic Effect Of Bilateral Subcostal Tap Block After Laparoscopic Cholecystectomy. J Ayub Med Coll Abbottabad, 30 (1): 12-5.

10.Saadati K, Razavi M, Nazemi S et al. (2016): Postoperative pain relief after laparoscopic cholecystectomy: intraperitoneal sodium bicarbonate versus normal saline. Gastroenterol Hepatol Bed Bench., 9 (3): 189-96.

11.Gad M, Dawood R, Antar M et al. (2019): Role of hysteroscopy and laparoscopy in evaluation of unexplained infertility. Menoufia Med J., 32: 1401-5.

12. Tsai H, Chen Y, Ho C et al. (2011): Maneuvers to decrease laparoscopy-induced shoulder and upper abdominal pain: a randomized controlled study. Arch Surg., 146 (12): 1360-6.

13.Kietpeerakool C, Rattanakanokchai S, Yantapant A et al. (2020): Pulmonary Recruitment Maneuver for Reducing Shoulder Pain after Laparoscopic Gynecologic Surgery: A Network Meta-Analysis of Randomized 
Controlled Trials. Minim Invasive Surg.,doi: 10.1155/2020/7154612.

14. Sao C, Chan-Tiopianco M, Chung K et al. (2019):Pain after laparoscopic surgery: Focus on shoulder-tip pain after gynecological laparoscopic surgery. J Chin Med Assoc., 82 (11): 819-26

15.Golparvar M, Saghaei M, Matin G (2014):Effects of intra-operative end-tidal carbon dioxide levels on the rates of post-operative complications in adults undergoing general anesthesia for percutaneous nephrolithotomy: A clinical trial. Advanced Biomedical Research, 3: 84.

16.Binda M (2015): Humidification during laparoscopic surgery: overview of the clinical benefits of using humidified gas during laparoscopic surgery. Arch Gynecol Obstet., 5: 955-71

17. Vandijk J, Dedden S, Geomini P et al. (2017): Post laparoscopic Reduction of pain By combining intraperitoneal normal salinE And the pulmonary Recruitment maneuver (POLAR BEAR trial). RCT to estimate reduction in pain after laparoscopic surgery when using a combination therapy of intraperitoneal normal saline and the pulmonary recruitment maneuver. BMC Womens Health, 17 (1): 42.

18.Balayssac D, Pereira B, Bazin J et al. (2017): Warmed and humidified carbon dioxide for abdominal laparoscopic surgery: meta-analysis of the current literature. Surg Endosc., 1: 1-12.

19. Ghauri S, Javaeed A, Mustafa $K$ et al. (2019):Bicarbonate Therapy for Critically Ill Patients with Metabolic Acidosis: A Systematic Review. Cureus, 11 (3): 42-97.

20. Velissaris D, Karamouzos V, Ktenopoulos $\mathbf{N}$ et al. (2015):The Use of Sodium Bicarbonate in the Treatment of Acidosis in Sepsis: A Literature Update on a Long Term Debate. doi: 10.1155/2015/605830.

21.Frank S, Lalonde D (2012): How acidic is the lidocaine we are injecting, and how much bicarbonate should we add? Can J Plast Surg., 20 (2): 71-3.

22. Carvalho B, Fuller A, Brummel C et al. (2007):Local infiltration of epinephrine-containing lidocaine with bicarbonate reduces superficial bleeding and pain during labor epidural catheter insertion: a randomized trial. Int $\mathbf{J}$ Obstet Anesth., 16 (2): 116-21.

23. Cepeda M, Tzortzopoulou A, Thackrey M et al. (2010): Adjusting the $\mathrm{pH}$ of lidocaine for reducing pain on injection. Cochrane Database Syst Rev., 12: 65-81.

24. Binda M (2015): Humidification during laparoscopic surgery: overview of the clinical benefits of using humidified gas during laparoscopic surgery. Arch Gynecol Obstet., 292 (5): 955-71.

25.Barczyński M, Herman $R$ (2004): Low-pressure pneumoperitoneum combined with intraperitoneal saline washout for reduction of pain after laparoscopic cholecystectomy: a prospective randomized study. Surg Endosc., 18: 1368-73.

26. Esmat M, Elsebae M, Nasr M et al. (2006):Combined low pressure pneumoperitoneum and intraperitoneal infusion of normal saline for reducing shoulder tip pain following laparoscopic cholecystectomy. World J Surg., 11: 1969-73

27.Seo Y, Lee H, Ha T et al. (2012):Effect of normal saline irrigation on attenuation of shoulder tip pain and on $\beta$ endorphin levels after laparoscopic cholecystectomy. J Laparoendosc Adv Surg Tech A., 4: 311-4.

28. Brokelman W, Lensvelt M, Borel-Rinkes I et al. (2011):Peritoneal changes due to laparoscopic surgery. Surg Endosc., 1: 1-9. 